\title{
Newton-Raphson method for accuracy enhancement of electro-optical targeting system
}

\author{
S.-T. Lu \\ C. Chou \\ Y.-P. Wu \\ H.-J. King
}

Indexing terms: Newton-Raphson algorithm. Targeting system

Abstract: A Newton-Raphson algorithm for optimising dimension parameters of the electrooptical targeting system (EOTS) is proposed. Those spatial parameters are critical to the accuracy of position measurement in the targeting system. To calibrate EOTS, the Newton-Raphson iteration method is used to enhance the system accuracy. This algorithm is based on four tested points where each point is required to be located in four different quadrants in the target area. The Newton-Raphson iteration method minimises the difference between the calculated and the measured values obtained from any of two tested locations. This algorithm enhances the system accuracy up to $\pm 1 \mathrm{~mm}$ within a $1 \mathrm{~m} \times 1 \mathrm{~m}$ targeting area.

\section{Introduction}

Different electro-optical targeting systems [1-4] have been proposed for measuring the speed and position of projectile rapidly and simultaneously. All of these systems have the advantages of real time measurement over the conventional method [5]. To keep the system accuracy, each of these systems must be calibrated before being tested. However, different systems have different calibration algorithms. Recently, S.T. Lu et al. [6] introduced an electro-optical targeting system (EOTS) by using lasers and cylindrical reflectors. The system accuracy on position measurement is based on spatial parameters of the main frame structure which we designed and measured. In general, the space between photodiodes needs to be measured accurately at the beginning, but the dimensions of the targeting area are more or less different from the corrected dimensions which need to be calibrated. It is because the accuracy of position measurement is critical to those spatial parameters that the Newton-Raphson iteration method was then suggested for use in this calibration process. There are four tested

(O) IEE, 1994

Paper 9697A (E13), first received 15th February and in revised form 26th May 1993

S.-T. Lu and Y.P. Wu is with the Department of Electrical Engineering, National Taiwan University, Taipei, Taiwan, Republic of China

C. Chou is with the School of Medical Technology, National Yang Ming Medical College, Taipei, Taiwan, Republic of China

H.-I. King is with the Department of Applied Physics, Chung-Cheng Institute of Technology, Ta-Shi, Taoyuan, Taiwan, Republic of China points which have to be located in different quadrants within the targeting area (see Section 4 and Fig. 3). Using this algorithm, we minimised the difference between the calibrated and the measured value obtained from any of two tested locations. After this algorithm, a system accuracy of EOTS better than $\pm 1 \mathrm{~mm}$ within a $1 \mathrm{~m} \times 1 \mathrm{~m}$ targeting area was reached.

\section{Operational principle of EOTS}

A simplified scheme of an electro-optical target system is presented in Fig. 1. A more physical structure is drawn in Fig. 2 as a reference. In Fig. 1, the numerical number in each small block represents a photodiode on an L-shaped photodiode array which has a total number of $2 \mathrm{~N}$ photodiodes. The small dot on the bottom line represents a cylindrical mirror from which fan-shaped laser beams emanate. The fan-shaped beams are directed to the Lshaped photodiode array to form an optical gate. EOTS uses two optical gates which are arranged on opposite sides of EOTS body to construct a two-dimensional position measurement system. For illustration clarity, the

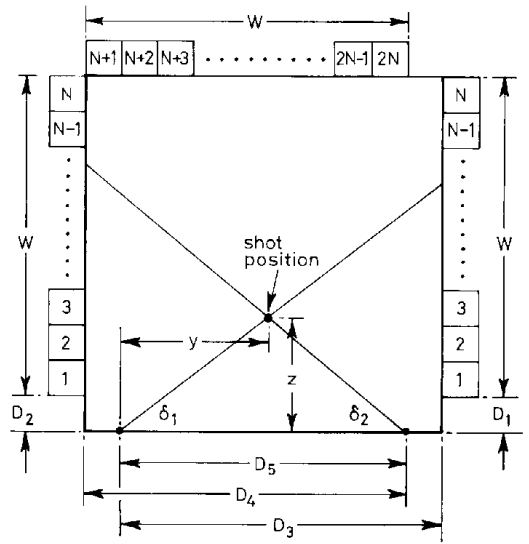

Fig. 1 Simplified scheme of electro-optical target system

This work was partially supported by the $\mathrm{Na}$ tional Science Council of ROC under the contract number NSC82-0404-E-010-012.

IEE Proc.-Sci. Meas. Technol, Vol. 141, No. 3, May 1994 
upper portion of $\mathrm{L}$-shaped photodiode array on the right side of EOTS is not shown. Now, consider a projectile normal incident upon the two optical gates. A portion of fan-shaped beams will be blocked by the projectile, and

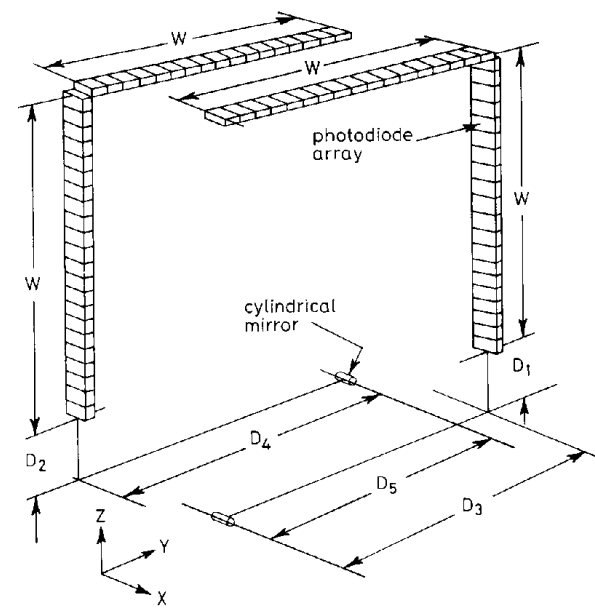

Fig. 2 Illustration of spatial parameters in electro-optical target

the respective photodiodes will be activated by the disturbance. In the first optical gate, the angle that relates to a photodiode, numbered $n$, can be calculated as

$$
\delta_{1}(n)=\arctan \frac{\frac{\left(n-\frac{1}{2}\right)}{N} W+D_{1}}{D_{3}} \text { for } 1 \leqslant n \leqslant N
$$

and

$$
\delta_{1}(n)=\arctan \frac{D_{1}+W}{D_{3}-\left(\frac{n-\frac{1}{2}}{N}-1\right) W}
$$

for $N+1 \leqslant n \leqslant 2 N \quad$ (2)

If certain photodiodes, numbered from $j$ to $k$, are activated by the projectile, the shot-position angle $\delta_{1}$ is given by

$$
\delta_{1}=\frac{\delta_{1}(j)+\delta_{1}(k)}{2}
$$

Similarly, the shot-position angle of the second optical gate $\delta_{2}$ is decided, and the shot-position of projectile is deduced in Cartesian coordinates as

$$
\begin{aligned}
& y=\frac{D_{5} \tan \delta_{2}}{\tan \delta_{1}+\tan \delta_{2}} \\
& \text { and } \\
& z=y \tan \delta_{1}
\end{aligned}
$$

where $W$ is the total length of $N$ photodiodes on one side of an optical gate, and $D_{1}$ to $D_{5}$ are the spatial parameters, defined in Fig. 1 and Fig. 2, about which we are specially concerned.

IEE Proc.-Sci. Meas. Technol., Vol. 141, No. 3, May 1994
3 Influence of the spatial parameters on system accuracy

The spatial parameters of EOTS are inevitably changed from the original designed values when the system is assembled. These changes will cause errors on position measurement of the projectile because those errors are critically related to the accuracy of position measurement in the targeting area of the EOTS. Fig. 3 shows a simplified front view of the EOTS. An area surrounded by a dashed line in the Figure is defined as the targeting area. The projectile is considered normally incident onto this area. As shown in Fig. 3, the target area is divided into four quadrants by two crossed lines. Each line is drawn from a cylindrical mirror (the small dot on the bottom line) to the opposite corner which corresponds to the corner of L-shaped photodiode array.

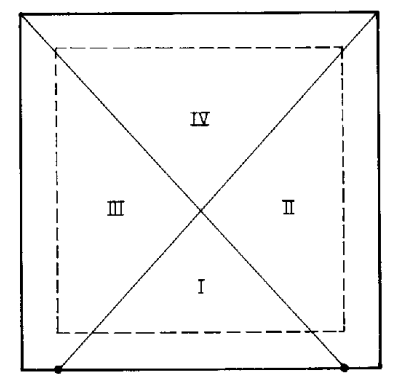

Fig. 3 Targeting area, surrounded by dash lines, is ditided into four quadrant

Small dots on bottom line represent cylindrical mirrors

Now, considering the influences caused by those parameters changes in the first quadrant; the tested location of a projectile that directs into this quadrant can be deduced in Cartesian coordinates as

$$
y=\frac{D_{3} D_{5}\left(A_{2}+D_{2}\right)}{D_{4}\left(A_{1}+D_{1}\right)+D_{3}\left(A_{2}+D_{2}\right)}
$$

and

$$
z=\frac{D_{5}\left(A_{1}+D_{1}\right)\left(A_{2}+D_{2}\right)}{D_{4}\left(A_{1}+D_{1}\right)+D_{3}\left(A_{2}+D_{2}\right)}
$$

where

$$
A_{1}=\frac{\left(n_{1}-\frac{1}{2}\right) W}{N}
$$

and

$$
A_{2}=\frac{\left(n_{2}-\frac{1}{2}\right) W}{N}
$$

The numbers $n_{1}$ and $n_{2}$ in eqns. 8 and 9 correspond to specific photodiodes which are related to the shot position angles $\delta_{1}$ and $\delta_{2}$, respectively. The values of $n_{1}$ and $n_{2}$ which are not necessarily integers will be located between 1 and $N$. Thus, the differential shot position wrt the differential spatial parameter $D_{1}$ can be found as

$$
\frac{\partial y}{\partial D_{1}}=-\frac{D_{3} D_{4} D_{5}\left(A_{2}+D_{2}\right)}{\left[D_{4}\left(A_{1}+D_{1}\right)+D_{3}\left(A_{2}+D_{2}\right)\right]^{2}}
$$


and

$$
\frac{\partial z}{\partial D_{1}}=\frac{D_{3} D_{5}\left(A_{2}+D_{2}\right)^{2}}{\left[D_{4}\left(A_{1}+D_{1}\right)+D_{3}\left(A_{2}+D_{2}\right)\right]^{2}}
$$

A plot of measured position variation against paramete $D_{1}$ change is shown in Fig. 4. Other equations about position variation in different quadrants wrt spatial parameter change can be found in the Appendix.

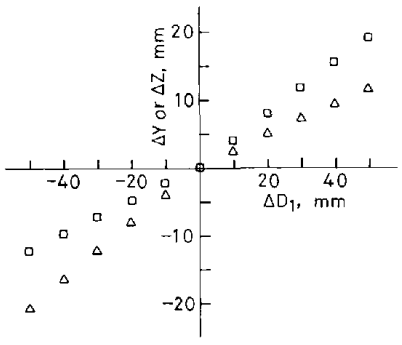

Fig. 4 Measured position tariation to parameter $D_{1}$ change $n_{1}$ is assumed to be 100 and $n_{2}$ also as 100 ; other parameters are: $W=1050$, are in mm; ' $\square \square \square$ ' represents $\Delta Y$ wrt $\Delta D_{1}$; ' $\Delta \Delta \Delta$ ' represents $\Delta Z$ wrt $\Delta D_{1}$

\section{Newton-Raphson correction algorithm}

We adopted the Newton-Raphson method [7] in the correction algorithm because of its fast convergence characteristics. At first, we set up a target disc right behind the second optical gate. Four bullets were shot. Each bullet was located at different quadrant in the targetin area. The positions of all activated photodiodes after each shot were recorded sequentially. After shooting, we measured all the distances $L_{i j}$ between any two of the four tested positions. $L_{i j}$ means the distance between position $i$ and position $j$ on the target disc. Five nonlinear equations of differences of $f_{i j}$ were obtained. They are

$$
f_{i j}=\left[\left(y_{i}-y_{j}\right)^{2}+\left(z_{i}-z_{j}\right)^{2}\right]^{1 / 2}-L_{i j}
$$

where $i$ is changed from 1 to 2 , and $j$ is from 2 to 4 , but $i \neq j . y$ and $z$ are calculated by substituting activated photodiode positions into eqns. 1 to 5 . Furthermore, the differential of eqn. 12 is deduced as

$$
\frac{\partial f_{i j}}{\partial D}=\frac{\left(y_{i}-y_{j}\right) \frac{\partial}{\partial D}\left(y_{i}-y_{j}\right)+\left(z_{i}-z_{j}\right) \frac{\partial}{\partial D}\left(z_{i}-z_{j}\right)}{\left[\left(y_{i}-y_{j}\right)^{2}+\left(z_{i}-z_{j}\right)^{2}\right]^{1 / 2}}
$$

From the Newton-Raphson method, we calculated $\Delta D$ which means increments by eqn. 14

$$
\left[\frac{\partial f_{i j}}{\partial D}\right][\Delta D]=-\left[f_{i j}\right]
$$

Therefore, the optimised spatial parameters $D^{\prime}=D+\Delta D$ of EOTS can be calculated by the iteration procedure where $D^{\prime}$ is the optimised spatial parameter and $D$ is the parameter before correction. The iteration processes keep going until $\sum\left(f_{i j}\right)^{2}$ is minimised. Finally, five spatia parameters $D_{1}^{\prime}$ to $D_{5}^{\prime}$ are precisely obtained. However, if any two of four calibrated positions are located in the same quadrant within the targeting area, the determinant of the Jacobian matrix $\left[\partial f_{i j} / \partial D\right]$ will be zero, which will force the computation of $\Delta D$ failure.

\section{Discussion and conclusions}

It is difficult to accurately measure the distances between any two of the tested positions in the targeting area during the calibration process. The distances between any two of different shot locations are necessary to be as large as possible to minimise the measurement error. Furthermore, the distance between the target and the muzzle should also be far enough for the projectile to be considered as normal incident upon the target area. Under these conditions, the Newton-Raphson method, which was used to solve five nonlinear equations to correct the spatial parameters, is a very effective method for calibrating EOTS. To obtain correct solutions, the initial value of the dimension of EOTS is very critical. Otherwise, the Newton-Raphson method will give incorrect solutions.

The algorithm has been used in a prototype system and enhanced its accuracy up to $\pm 1 \mathrm{~mm}$ within a $1 \mathrm{~m} \times 1 \mathrm{~m}$ targeting area.

\section{References}

1 CRITTENDEN, E.C., KING, R.A., and ANDREWS, T.C.: 'Target measurement system for precise projectile location'. US Patent 3727069, 1973

2 BAILEY, T.B., and BATES, J.: 'Electro-optical projectile analyzer' US Patent 4272189, 1981

DECK, L.L.: 'An optical device for rapid measurement of the speed, dispersion, attack angle and shock wave of high velocity small caliber projectiles'. Proceedings of the tenth international symposium on Ballistics, 1987, 1, pp. 1-9

4 LU, S.T, YU, A.T, and CHOU, C. 'Electro-optics target for position and speed measurement'. Proceedings of SPIE, 1988, 981, 250-254

5 BETTERMANN, P., and MAYER, F.: 'Handbook on weaponry' (Rheinmetall GmbH, Dusseldorf, 1982)

6 LU, S.T., CHOU, C., LEE, M.C., and WU, Y.P.: 'Electro-optical target system for position and speed measurement', IEE Proc A, 1993,140 , pp. 252256

7 WISMER, D.A., and CHATTERGY, R.: 'Introduction to nonlinear optimization: a problem solving approach' (North Holland Publishing Company, 1978)

\section{Appendix}

Let $n_{1}$ and $n_{2}$ be the number of corresponding photodiodes on the first and the second optical gate, which is also related to the shot position angle $\delta_{1}$ and $\delta_{2}$, respectively. Here, we define

$$
\begin{aligned}
& A_{1}=\frac{\left(n_{1}-\frac{1}{2}\right) W}{N} \\
& A_{2}=\frac{\left(n_{2}-\frac{1}{2}\right) W}{N} \\
& A_{3}=\left(2 N+\frac{1}{2}-n_{1}\right) \frac{W}{N}
\end{aligned}
$$

and

$$
A_{4}=\left(2 N+\frac{1}{2}-n_{2}\right) \frac{W}{N}
$$

In the first quadrant, where $1 \leqslant n_{1} \leqslant N$ and $1 \leqslant n_{2} \leqslant N$, we have the following equations:

$$
\begin{aligned}
& y=\frac{D_{3} D_{5}\left(A_{2}+D_{2}\right)}{K} \\
& z=\frac{D_{5}\left(A_{1}+D_{1}\right)\left(A_{2}+D_{2}\right)}{K}
\end{aligned}
$$

IEE Proc.-Sci. Meas. Téchnol., Vol. 141, No. 3, May 1994 


$$
\begin{aligned}
& \frac{\partial y}{\partial D_{1}}=-\frac{D_{3} D_{4} D_{5}\left(A_{2}+D_{2}\right)}{K^{2}} \\
& \frac{\partial y}{\partial D_{2}}=\frac{D_{3} D_{4} D_{5}\left(A_{1}+D_{1}\right)}{K^{2}} \\
& \frac{\partial y}{\partial D_{3}}=\frac{D_{4} D_{5}\left(A_{1}+D_{1}\right)\left(A_{2}+D_{2}\right)}{K^{2}} \\
& \frac{\partial y}{\partial D_{4}}=-\frac{D_{3} D_{5}\left(A_{1}+D_{1}\right)\left(A_{2}+D_{2}\right)}{K^{2}} \\
& \frac{\partial y}{\partial D_{5}}=\frac{D_{3}\left(A_{2}+D_{2}\right)}{K} \\
& \frac{\partial z}{\partial D_{1}}=\frac{D_{3} D_{5}\left(A_{2}+D_{2}\right)^{2}}{K^{2}} \\
& \frac{\partial z}{\partial D_{2}}=\frac{D_{4} D_{5}\left(A_{1}+D_{1}\right)^{2}}{K^{2}} \\
& \frac{\partial z}{\partial D_{3}}=-\frac{D_{5}\left(A_{1}+D_{1}\right)\left(A_{2}+D_{2}\right)^{2}}{K^{2}} \\
& \frac{\partial z}{\partial D_{4}}=-\frac{D_{5}\left(A_{1}+D_{1}\right)^{2}\left(A_{2}+D_{2}\right)}{K^{2}} \\
& \frac{\partial z}{\partial D_{5}}=\frac{\left(A_{1}+D_{1}\right)\left(A_{2}+D_{2}\right)}{K} \\
& \text { where } \\
& K=D_{4}\left(A_{1}+D_{1}\right)+D_{3}\left(A_{2}+D_{2}\right)
\end{aligned}
$$

In the second quadrant where $1 \leqslant n_{1} \leqslant N$ and $N+1 \leqslant$ $n_{2} \leqslant 2 N$, we have the following equations:

$$
\begin{aligned}
& y=\frac{D_{3} D_{5}\left(D_{2}+W\right)}{S} \\
& z=\frac{D_{5}\left(A_{1}+D_{1}\right)\left(D_{2}+W\right)}{S} \\
& \frac{\partial y}{\partial D_{1}}=-\frac{D_{3} D_{5}\left(D_{2}+W\right)\left(A_{4}+D_{4}-W\right)}{S^{2}} \\
& \frac{\partial y}{\partial D_{2}}=\frac{D_{3} D_{5}\left(A_{1}+D_{1}\right)\left(A_{4}+D_{4}-W\right)}{S^{2}} \\
& \frac{\partial y}{\partial D_{3}}=\frac{D_{5}\left(A_{1}+D_{1}\right)\left(D_{2}+W\right)\left(A_{4}+D_{4}-W\right)}{S^{2}} \\
& \frac{\partial y}{\partial D_{4}}=-\frac{D_{3} D_{5}\left(A_{1}+D_{1}\right)\left(D_{2}+W\right)}{S^{2}} \\
& \frac{\partial y}{\partial D_{5}}=\frac{D_{3}\left(D_{2}+W\right)}{S} \\
& \frac{\partial z}{\partial D_{1}}=\frac{D_{3} D_{5}\left(D_{2}+W\right)^{2}}{S^{2}} \\
& \frac{\partial z}{\partial D_{2}}=\frac{D_{5}\left(A_{1}+D_{1}\right)^{2}\left(A_{4}+D_{4}-W\right)}{S^{2}} \\
& \frac{\partial z}{\partial D_{3}}=-\frac{D_{5}\left(D_{2}+W\right)^{2}\left(A_{1}+D_{1}\right)}{S^{2}} \\
& \frac{\partial z}{\partial D_{4}}=-\frac{D_{5}\left(D_{2}+W\right)\left(A_{1}+D_{1}\right)^{2}}{S^{2}} \\
& \frac{\partial z}{\partial D_{5}}=\frac{\left(D_{2}+W\right)\left(A_{1}+D_{1}\right)}{S}
\end{aligned}
$$

IEE Proc.-Sci. Meas. Technol., Vol. 14I, No. 3, May 1994

$(40)$ where

$$
S=\left(A_{1}+D_{1}\right)\left(A_{4}+D_{4}-W\right)+D_{3}\left(D_{2}+W\right)
$$

In the third quadrant where $N+1 \leqslant n_{1} \leqslant 2 N$ and $1 \leqslant$ $n_{2} \leqslant N$, we have the following equations:

$$
y=\frac{D_{5}\left(A_{2}+D_{2}\right)\left(A_{3}+D_{3}-W\right)}{U}
$$$$
z=\frac{D_{5}\left(D_{1}+W\right)\left(A_{2}+D_{2}\right)}{U}
$$

$$
\frac{\partial y}{\partial D_{1}}=-\frac{D_{4} D_{5}\left(A_{3}+D_{3}-W\right)\left(A_{2}+D_{2}\right)}{U^{2}}
$$

$$
\frac{\partial y}{\partial D_{2}}=\frac{D_{4} D_{5}\left(D_{1}+W\right)\left(A_{3}+D_{3}-W\right)}{U^{2}}
$$

$\frac{\partial y}{\partial D_{3}}=\frac{D_{4} D_{5}\left(D_{1}+W\right)\left(A_{2}+D_{2}\right)}{U^{2}}$

$\frac{\partial y}{\partial D_{4}}=-\frac{D_{5}\left(D_{1}+W\right)\left(A_{3}+D_{3}-W\right)\left(A_{2}+D_{2}\right)}{U^{2}}$

$\frac{\partial y}{\partial D_{5}}=\frac{\left(A_{2}+D_{2}\right)\left(A_{3}+D_{3}-W\right)}{U}$

$\frac{\partial z}{\partial D_{1}}=\frac{D_{5}\left(A_{2}+D_{2}\right)^{2}\left(A_{3}+D_{3}-W\right)}{U^{2}}$

$\frac{\partial z}{\partial D_{2}}=\frac{D_{4} D_{5}\left(D_{1}+W\right)^{2}}{U^{2}}$

$\frac{\partial z}{\partial D_{3}}=-\frac{D_{5}\left(D_{1}+W\right)\left(A_{2}+D_{2}\right)^{2}}{U^{2}}$

$\frac{\partial z}{\partial D_{4}}=-\frac{D_{5}\left(D_{1}+W\right)^{2}\left(A_{2}+D_{2}\right)}{U^{2}}$

$\frac{\partial z}{\partial D_{5}}=\frac{\left(D_{1}+W\right)\left(A_{2}+D_{2}\right)}{U}$

where

$$
U=D_{4}\left(D_{1}+W\right)+\left(A_{3}+D_{3}-W\right)\left(A_{2}+D_{2}\right)
$$

In the fourth quadrant, where $N+1 \leqslant n_{1} \leqslant 2 N$ and $N$ $+1 \leqslant n_{2} \leqslant 2 N$, we have the following equations:

$$
y=\frac{D_{5}\left(D_{2}+W\right)\left(A_{3}+D_{3}-W\right)}{V}
$$

$$
z=\frac{D_{5}\left(D_{1}+W\right)\left(D_{2}+W\right)}{V}
$$
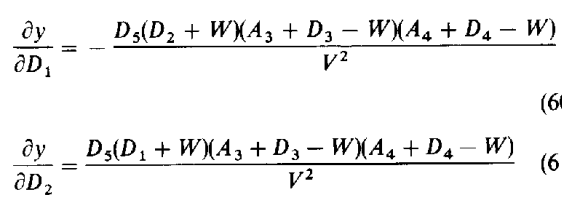

$\frac{\partial y}{\partial D_{3}}=\frac{D_{5}\left(D_{1}+W\right)\left(D_{2}+W\right)\left(A_{4}+D_{4}-W\right)}{V^{2}}$$$
\frac{\partial y}{\partial D_{4}}=-\frac{D_{5}\left(D_{1}+W\right)\left(D_{2}+W\right)\left(A_{3}+D_{3}-W\right)}{V^{2}}
$$

$\frac{\partial y}{\partial D_{5}}=\frac{\left(D_{2}+W\right)\left(A_{3}+D_{3}-W\right)}{V}$ 


$$
\begin{aligned}
& \frac{\partial z}{\partial D_{1}}=\frac{D_{5}\left(D_{2}+W\right)^{2}\left(A_{3}+D_{3}-W\right)}{V^{2}} \\
& \frac{\partial z}{\partial D_{2}}=\frac{D_{5}\left(D_{1}+W\right)^{2}\left(A_{4}+D_{4}-W\right)}{V^{2}} \\
& \frac{\partial z}{\partial D_{3}}=-\frac{D_{5}\left(D_{1}+W\right)\left(D_{2}+W\right)^{2}}{V^{2}}
\end{aligned}
$$

$\frac{\partial z}{\partial D_{4}}=-\frac{D_{5}\left(D_{1}+W\right)^{2}\left(D_{2}+W\right)}{V^{2}}$

(66)

$\frac{\partial z}{\partial D_{5}}=\frac{\left(D_{1}+W\right)\left(D_{2}+W\right)}{V}$

where

(67)

$V=\left(D_{1}+W\right)\left(A_{4}+D_{4}-W\right)$

$+\left(D_{2}+W\right)\left(A_{3}+D_{3}-W\right)$

(70) 